\title{
Genome-wide DNA methylation analysis reveals that mouse chemical iPSCs have closer epigenetic features to mESCs than OSKM-integrated iPSCS
}

\author{
Wangfang Ping ${ }^{1,2,3}$, Jian $\mathrm{Hu}^{4}$, Gongcheng $\mathrm{Hu}^{1,3}$, Yawei Song ${ }^{1,3}$, Qing Xia ${ }^{1,3}$, Mingze Yao ${ }^{1,3}$, Shixin Gong ${ }^{1,3}$,
} Cizhong Jiang (iD ${ }^{4}$ and Hongjie Yao ${ }^{1,3}$

\begin{abstract}
Induced pluripotent stem cells can be derived from somatic cells through ectopic expression of transcription factors or chemical cocktails. Chemical iPSCs (C-iPSCs) and OSKM-iPSCs (4F-iPSCs) have been suggested to have similar characteristics to mouse embryonic stem cells (mESCs). However, their epigenetic equivalence remains incompletely understood throughout the genome. In this study, we have generated mouse C-iPSCs and 4F-iPSCs, and further compared the genome-wide DNA methylomes of C-iPSCs, 4F-iPSCs, and mESCs that were maintained in $2 i$ and LIF. Three pluripotent stem cells tend to be low methylated overall, however, DNA methylations in some specific regions (such as retrotransposons) are cell type-specific. Importantly, C-iPSCs are more hypomethylated than 4F-iPSCs. Bisulfite sequencing indicated that DNA methylation status in several known imprinted clusters, such as: DIk1-Dio3 and Peg12Ube3a, in C-iPSCs are closer to those of mESCs than 4F-iPSCs. Overall, our data demonstrate the reprogramming methods-dependent epigenetic differences of C-iPSCs and 4F-iPSCs and reveal that C-iPSCs are more hypomethylated than OSKM-integrated iPSCs.
\end{abstract}

\section{Introduction}

Embryonic stem cells (ESCs) and induced pluripotent stem cells (iPSCs) have great therapeutic potential for regenerative medicine and new drug screening ${ }^{1}$. Induced pluripotent stem cells can be achieved by several techniques, such as somatic cell nuclear transfer, cell fusion, exogenous transfection of transcriptional factors, and small-molecule treatment ${ }^{2-5}$. However, there are

Correspondence: Cizhong Jiang (czjiang@tongji.edu.cn) or

Hongjie Yao (yao_hongjie@gibh.ac.cn)

${ }^{1}$ CAS Key Laboratory of Regenerative Biology, Joint School of Life Sciences,

CAS Center for Excellence in Molecular Cell Science, Guangzhou Institutes of Biomedicine and Health, Chinese Academy of Sciences, Guangzhou Medical University, Guangzhou, China

${ }^{2}$ Institute of Health Sciences, Anhui University, Hefei, China

Full list of author information is available at the end of the article

Wangfang Ping and Jian Hu have contributed equally to this work.

Edited by $Y$ Shi concerns that reprogramming may introduce subtle specific defects that could impact the safety of iPSCs ${ }^{6,7}$. Similar arguments continue as to whether these reprogrammed cells are completely equivalent in both function and character to genuine ESCs ${ }^{8}$. Previous studies have shown that the global expression patterns and epigenetic modifications were very similar between ESCs and 4Finduced iPSCs ${ }^{9,10}$, but there are yet some key differences, such as 4F-iPSCs have a unique gene expression signature, including microRNAs (miRNAs) and long noncoding RNAs ${ }^{11,12}$. Besides, 4F-iPSCs have genomic copynumber variations ${ }^{13}$. And the reactivation of $\mathrm{c}-\mathrm{Myc}$ can cause tumor formation in chimeric mice derived from 4FiPSCs $^{14}$.

Epigenetic differences have also been observed between 4F-iPSCs and ESCs. DNA methylation is an important

\section{(c) The Author(s) 2018}

(c) (i) Open Access This article is licensed under a Creative Commons Attribution 4.0 International License, which permits use, sharing, adaptation, distribution and reproduction (c) in any medium or format, as long as you give appropriate credit to the original author(s) and the source, provide a link to the Creative Commons license, and indicate if changes were made. The images or other third party material in this article are included in the article's Creative Commons license, unless indicated otherwise in a credit line to the material. If material is not included in the article's Creative Commons license and your intended use is not permitted by statutory regulation or exceeds the permitted use, you will need to obtain permission directly from the copyright holder. To view a copy of this license, visit http://creativecommons.org/licenses/by/4.0/. 
a

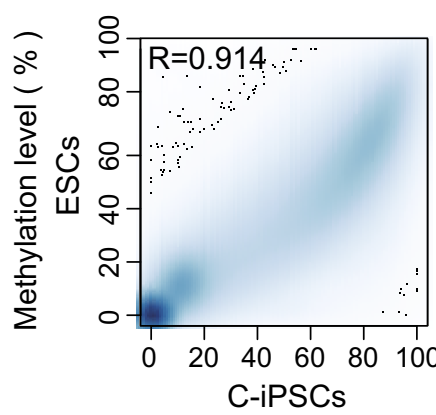

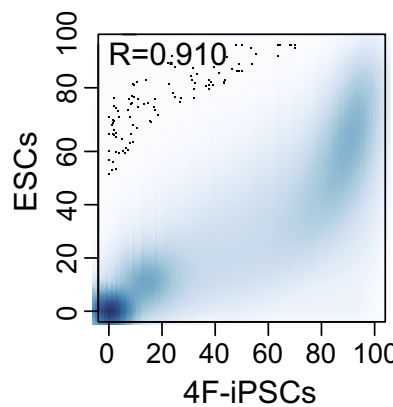

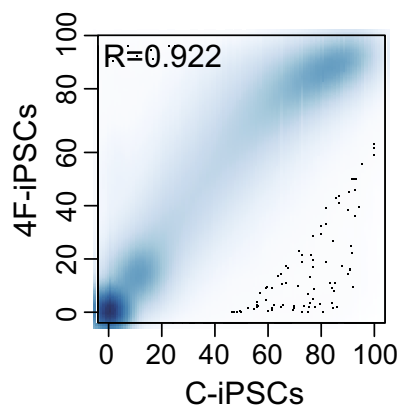

Methylation level ( \% ) b

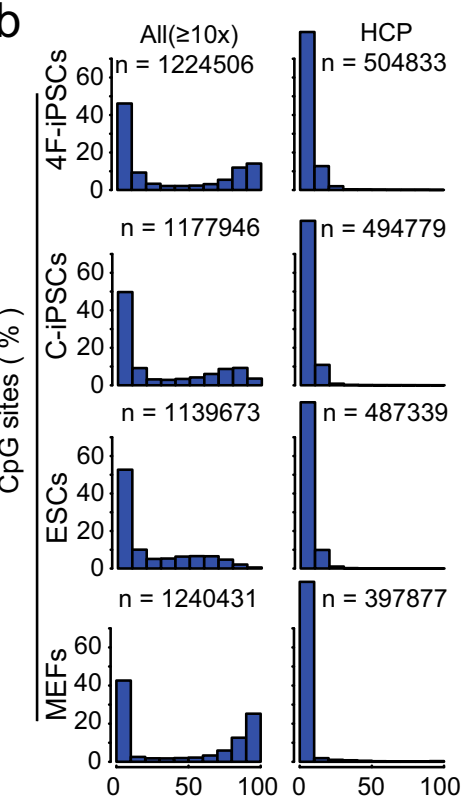

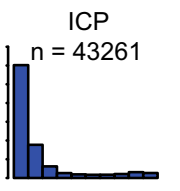
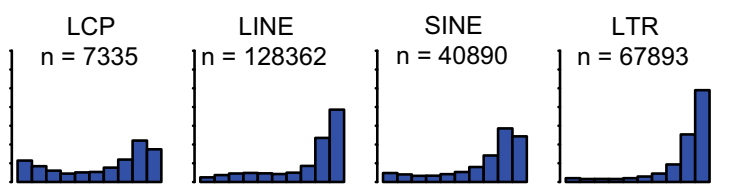

Others
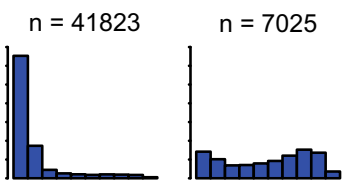

$n=120183$
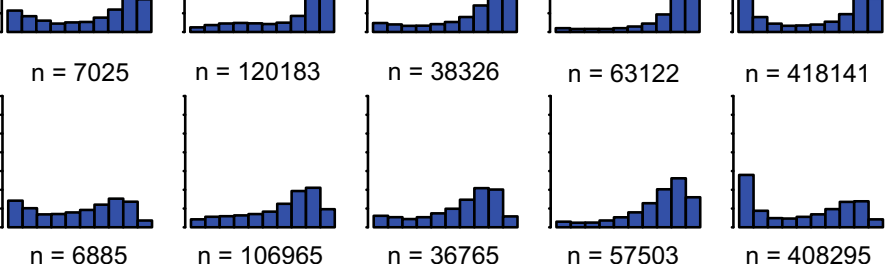

$\mathrm{n}=106965$

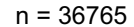

$\mathrm{n}=57503$

$n=408295$
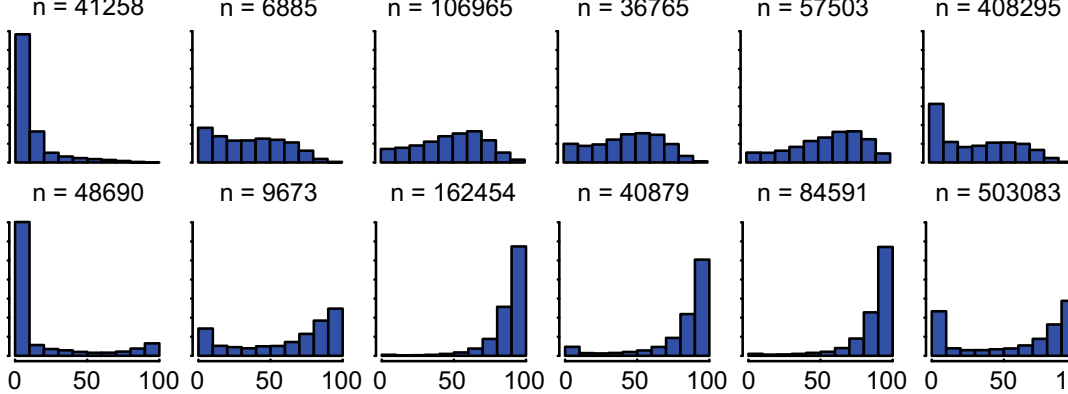

Methylation level ( \% )

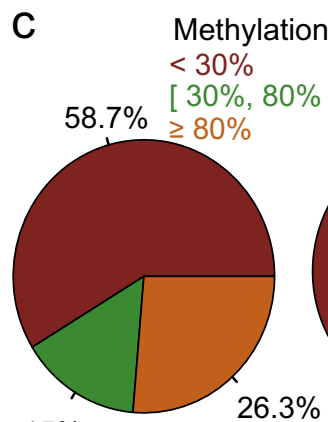

$15 \%$
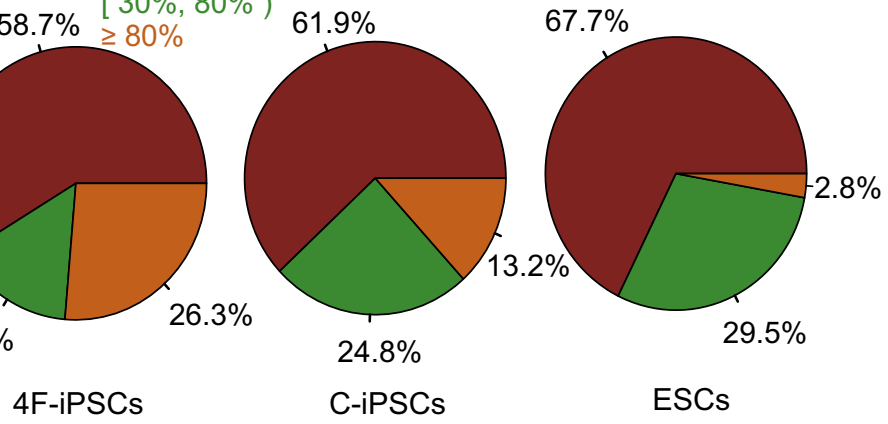

Fig. 1 Profiles of CpG methylation in mESCs, C-iPSCs, and 4F-iPSCs for CpGs with $\geq 10$-fold coverage. a Density scatter plots of CpG methylation levels between mESCs, C-iPSCs, and 4F-iPSCs. Spearman's $R$ is indicated. $\mathbf{b}$ Histograms showing the distribution of CpG methylation levels (\%) in mESCs, C-iPSCs, 4F-iPSCs, and MEFs across the entire genome (All), promoters with different CpG density (HCPs, ICPs, and LCPs), interspersed repeat elements (LINEs, SINEs, and LTRS) and other genomic regions excluding promters and LINEs, SINEs, and LTRs. c Pie charts representing the frequency of CpGs grouped by methylation levels 
epigenetic modification in regulation of gene expression, genomic stability, $\mathrm{X}$ chromosome inactivation, and genomic imprinting. It is regarded as an obstacle in somatic cell reprogramming ${ }^{15,16}$, and there are strong hints that DNA methylation is altered in 4F-iPSCs. For example, differential DNA methylation distinguishes human iPSCs and hESCs ${ }^{6,17}$. DNA hypermethylation in 4F-iPSCs reduces their ability to differentiate toward a hematopoietic cell fate ${ }^{18}$. Similarly, abnormal DNA hypermethylation in 4F-iPSCs leads to aberrant silencing at the Dlk1-Dio3 imprinted gene cluster, which decreases the developmental potential of 4F-iPSCs into chimaeric and $4 \mathrm{n}$ complementation-competent-iPSCs mice ${ }^{19}$. These epigenetic abnormalities remain a concern for the utility and safety of 4F-iPSCs in regenerative and clinical medicine.

Small chemical molecules can regulate cell signaling pathways and the epigenetic status of cells to control cell fates $^{20}$. For example, the small molecules valproic acid (VPA, a histone deacetylase inhibitor) and vitamin C (Vc) have both been reported to prevent the abnormal silencing of the Dlk1-Dio3 locus in 4F-iPSCs ${ }^{19,21}$. Chemical iPSCs (C-iPSCs) ${ }^{4,22,23}$ and 4F-iPSCs have been suggested to have similar characteristics to mouse embryonic stem cells (mESC). However, their epigenetic equivalence remains incompletely unclear.

Here we investigated epigenetic DNA methylation features of three different pluripotent stem cells (C-iPSCs, 4F-iPSCs, and mESCs). Our results reveal that C-iPSCs are globally hypomethylated compared to 4F-iPSCs and the DNA methylation status of imprinted regions of mESCs is closer to that of C-iPSCs than 4F-iPSCs.

\section{Results \\ C-iPSCs, 4F-iPSCs, and mESCs all share a similar DNA methylome globally}

To profile DNA methylation in C-iPSCs, we first generated C-iPSCs (Supplementary Figure 1a) from mouse embryonic fibroblasts (MEFs) as previously reported ${ }^{22}$. The established C-iPSC lines expressed high levels of pluripotency marker genes, such as Oct4 and Nanog (Supplementary Figure 1b). The gene expression levels of pluripotency markers and DNA methylation status at Nanog promoters were similar among C-iPSCs, mESCs and 4F-iPSCs, which were induced by Yamanaka factors (Supplementary Figure 1c and d). To gain insight into the DNA methylomes of C-iPSCs, 4F-iPSCs, and mESCs, we performed reduced representation bisulfite sequencing $(\text { RRBS })^{24}$, which interrogated 1,106,981 CpG sites.

The global DNA methylation profiles were highly reproducible between replicates $(R>0.9$, Supplementary Figure 1e) and highly correlated between these cells derived using different methods $(R>0.922$, Fig. 1a). DNA methylation levels were generally low in all three pluripotent cell types compared with MEFs, but prominently bimodal in 4F-iPSCs (Fig. 1b). The bimodality was less prominent in C-iPSCs and was absent in mESCs (Fig. 1b). To explore this bimodality in detail, we broke down the CpG sites into different classes (Fig. 1b). The results indicated that the methylation levels of high-CpGdensity promoters (HCP) and intermediate-CpG-density promoters (ICP) were near-indistinguishable among the three pluripotent stem cells, even in MEFs, whereas the profiles in low-CpG-density promoters (LCP) were closer between mESCs and C-iPSCs than to 4F-iPSCs and MEFs (Fig. 1b). In addition, LCP methylations in both 4F-iPSCs and MEFs were bimodal (Fig. 1b). An important class of DNA that is methylated is the long/short-interspersed repeat elements (LINEs, SINEs) and long-terminal repeats (LTRs) that are generally suppressed by DNA methylation in somatic cells, and are mostly demethylated and active in both the reprogramming cells and $\mathrm{mESCs}^{25-27}$. Analysis of our DNA methylation data indicated a substantial difference in the methylation of LINEs, SINEs, and LTRs and they were all hypermethylated in 4F-iPSCs, which is similar to MEFs, but showed demetylation in mESCs and C-iPSCs (Fig. 1b).

We further examined the distribution of all CpGs grouped by methylation levels: high $(\geq 0.8)$, intermediate $(\geq 0.3$ and $<0.8)$, or low $(<0.3)$. There was no significant difference in intermediate methylation levels, but the low and high methylations showed cell-specificity, emphasizing this change in bimodality. Notably, 4F-iPSCs were globally hypermethylated compared to mESCs $(26.3 \%$ in 4F-iPSCs vs. $2.8 \%$ in mESCs) and C-iPSCs were in between (13.2\%) (Fig. 1c). Taken together, iPSCs and mESCs overall share a globally similar DNA methylome with highest portion of hypermethylated CpGs in 4FiPSCs, second in C-iPSCs, and least in mESCs.

\section{C-iPSCs are more DNA hypomethylated than 4F-iPSCs in a genome-wide scale}

We next identified 31,693 (about 3\% of all CpGs) and 157,365 (about 14\% of all CpGs) strongly hypermethylated CpG sites in C-iPSCs and 4F-iPSCs compared with mESCs, respectively (for strongly hypermethylated sites, the differences of methylation level were set as $>0.333$. Fisher's $t$ test $p$ value $<0.05$ according to Model-based Analysis of Bisulfite Sequencing data (MOABS)). Most of the strongly hypermethylated CpG sites were common between C-iPSCs and 4F-iPSCs (Fig. 2a). This suggested that mESCs were closer to C-iPSCs than 4F-iPSCs, at least from the perspective of the strongly hypermethylated CpGs. Comparison of the common 29,756 strongly hypermethylated CpGs showed that the DNA methylation levels were significantly higher in 4F-iPSCs than in CiPSCs (Fig. 2b). We further compared the DNA methylomes of 4F-iPSCs and C-iPSCs, and identified 14,749 
a

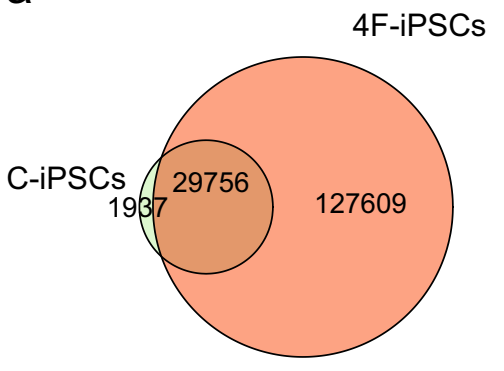

Strongly hypermethylated CpGs compared to ESCs b

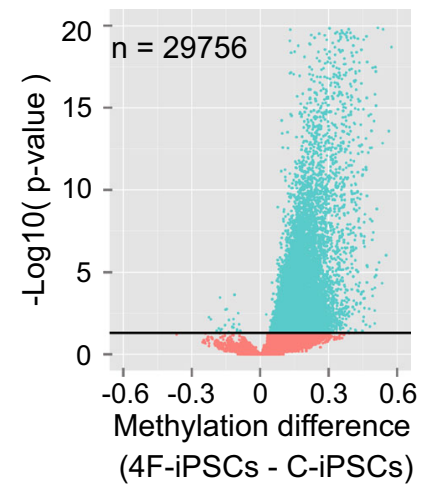

C

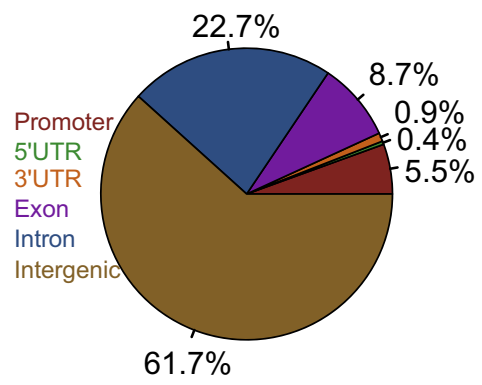

Strongly hypermethylated CpGs in 4F-iPSCs compared to C-iPSCs

Fig. 2 Comparison of DNA methylation levels between C-iPSCs and 4F-iPSCs. a Venn diagram showing the overlap between strong hypermethylated CpGs (33.3\% higher than mESCs) in C-iPSCs and 4F-iPSCs. b Volcano plot showing the differences in methylation level (4F-iPSCs-CiPSCs) of the common strong hypermethylated CpGs in C-iPSCs and 4F-iPSCs (the overlapped ones in a). $P$ values are calculated by Fisher's exact test. The black horizontal line indicates $p$ value $=0.05$. c Pie chart representing the strong hypermethylated CpGs in 4F-iPSCs compared to C-iPSCs (increased $\geq 33.3 \%$ ) with respect to their genomic locations

CpGs and 593 differentially methylated regions (DMRs) that were strongly hypermethylated in 4F-iPSCs, and only 311 CpGs and 21 DMRs that were strongly hypomethylated in 4F-iPSCs (Supplementary Figure 2a). This confirmed 4F-iPSCs had higher levels of DNA methylation compared to C-iPSCs. The strongly hypermethylated CpGs of each cell type were largely located in intergenic and intronic regions (Fig. 2c and Supplementary Figure $2 b$ ), in agreement with the bimodality seen in the LINEs/SINEs and LTRs (Fig. 1b), which are often intergenic.

\section{Promoter methylation levels in C-iPSCs but not in 4F-iPSCs resemble those in $\mathrm{mESCs}$}

DNA methylation of gene promoters is associated with chromatin configuration and gene expression. So we further compared DMRs of promoter regions among 4FiPSCs, C-iPSCs, and mESCs. Compared with mESCs, strongly hypermethylated DMRs were clustered into three classes (Fig. 3a). The class 1 showed that DNA methylation levels $(>80 \%)$ in C-iPSCs and 4F-iPSCs were both higher than the levels in mESCs. The class 2 showed that 4F-iPSCs (around 80\%) were hypermethylated compared to mESCs and C-iPSCs (around 50\%) were in between. The DNA methylation levels in class 3 of C-iPSCs were closer to mESCs compared with those in 4F-iPSCs. GO analysis indicated that the genes that have the similar DNA hypomethylation levels between C-iPSCs and mESCs were involved in chromosome organization during meiotic cell cycle, meiotic nuclear division, and synapsis (Fig. 3b).

To gain insights into the difference of promoter methylation levels between the induced pluripotent cell types, we profiled DNA methylation and compared the
DMRs of gene promoters between 4F-iPSCs and C-iPSCs. Clustering analysis showed that methylation levels in the DMRs of these promoters were similar between C-iPSCs and mESCs (Fig. 4a). Profiling analysis of the expression levels of the corresponding genes also clustered C-iPSCs and mESCs together (Fig. 4b). Moreover, most those genes that were repressed in 4F-iPSCs wereactivated in C-iPSCs and mESCs. Of particular interest is Impact, a gene preferentially expressed in neurons and modulating neurite outgrowth ${ }^{28}$. Impact was hypermethylated in 4FiPSCs but hypomethylated in C-iPSCs and mESCs (Fig. 4c), which were confirmed by bisulfite sequencing (BS-seq) (Fig. 4d). Quantitative Real-time Polymerase Chain Reaction (qRT-PCR) analysis showed that the expression of Impact was significantly decreased in $4 \mathrm{~F}$ iPSCs (Fig. 4e). In addition, we observed similar correlations between gene expression and methylation status in promoter DMRs in the imprinted Snrpn/Snurf loci (Supplementary Figure $3 \mathrm{a}-\mathrm{c}$ ). However, a small group of genes including Magel2, Trpc5, and so on showed a methylated promoter, but were expressed, while Prpf39 was demethylated in 4F-iPSCs but methylated in mESCs and $\mathrm{C}$-iPSCs and was corrsepondingly expressed only in 4F-iPSCs (Fig. 4a, b).

\section{The DNA methylation and transcription profiles of imprinted genes in C-iPSCs are more similar to mESCs than 4F-iPSCs}

We noticed that, among our list of DMR genes, there were several well-known imprinted genes, such as H19, Peg10, and Impact. The murine genome contains around 150 imprinted genes that are typically located in clusters $^{29}$. Importantly, imprinted genes have important roles in mammalian development ${ }^{30}$. Therefore, we next 


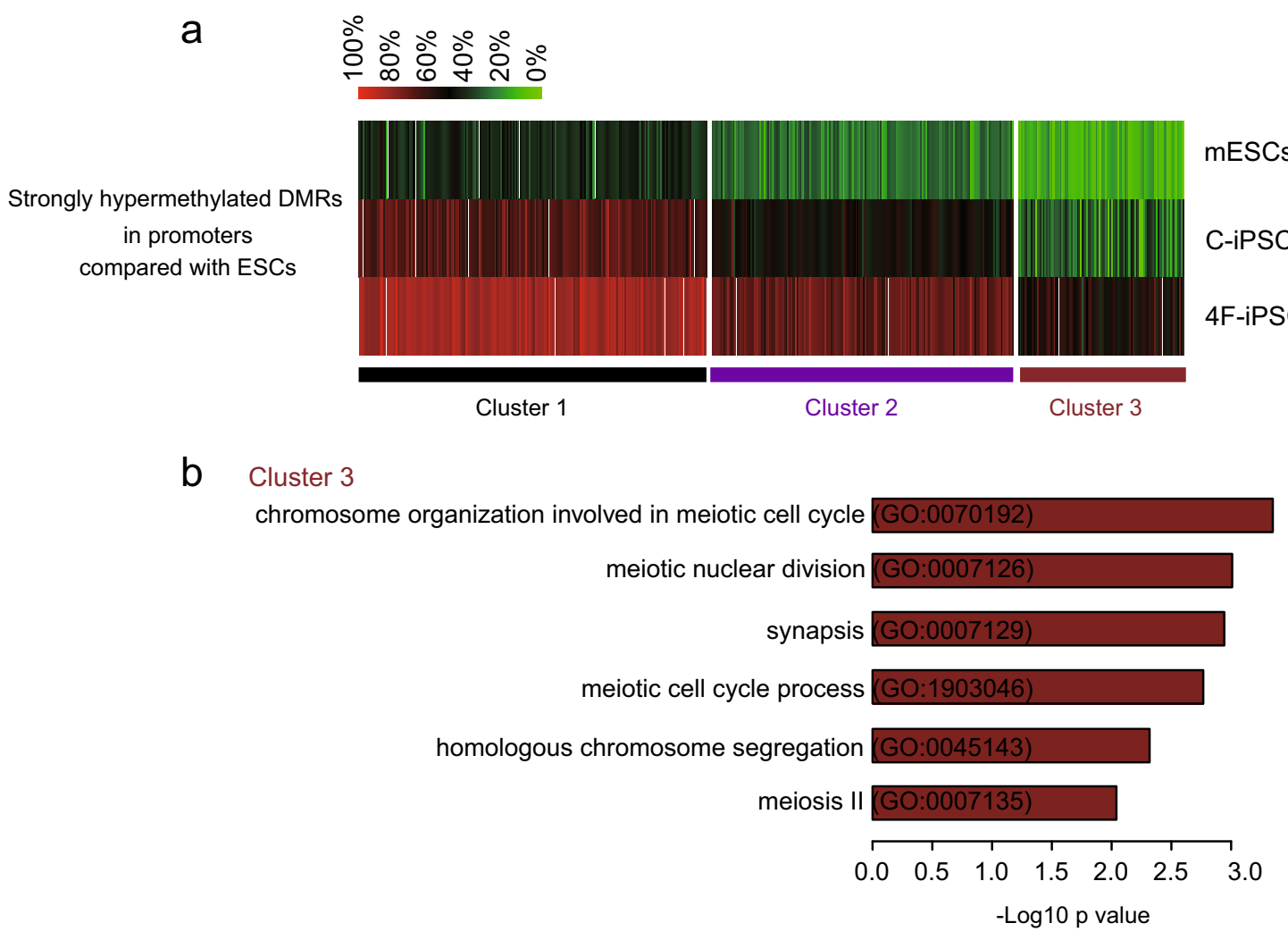

Fig. 3 Clustering of promoter-DMRs methylation levels among C-iPSCs, 4F-iPSCs, and mESCs. a A methylation ratio (\%) heatmap in each sample of all the strongly hypermethylated DMRs within promoter regions in C-iPSCs and 4F-iPSCs compared with mESCs. K-means $(K=3)$ clustering is performed. b Gene ontology (GO) terms associated with the genes within cluster 3 in a

focused on the DNA methylation and transcription levels of these imprinted genes. We calculated the methylation levels in the known imprinted clusters that spread over 20 $\mathrm{kb}-3.7 \mathrm{Mb}$ of $\mathrm{DNA}^{31}$, such as the Dlk1-Dio3, Peg12Ube3a, H19-Igf2, Mest-Copg2, Ddc-Grb10, Peg3-Usp9, Kcnq1ot1-Cdkn1c loci. The results indicated that mESCs and C-iPSCs showed similar hypomethylation levels in the DMRs, whereas 4F-iPSCs were hypermethylated (Fig. 5a). Consistently, expression profiles of the genes within these imprinting regions clustered mESCs and C-iPSCs together (Fig. 5b). The Dlk1-Dio3 imprinting cluster was hypermethylated in 4F-iPSCs. In contrast, this cluster was hypomethylated in C-iPSCs and mESCs (Fig. 5a, c). The cluster contains the intergenic germline-derived DMR (IG-DMR), whose methylation patterns are established in the germline. The IG-DMR, located $70 \mathrm{~kb}$ downstream of $D l k 1$ to $15 \mathrm{~kb}$ upstream of Gtl2, is believed to be a control element for this imprinted gene cluster ${ }^{32,33}$. The methylation levels of IG-DMR in C-iPSCs resembled those of mESCs, but those in 4F-iPSCs were the highest, which was confirmed by BS-seq (Fig. 5d). Correspondingly, the genes in Dlk1-Dio3 cluster were aberrantly silent in 4FiPSCs, including Meg3 and Mirg (Supplementary
Figure 4a and b). What's more, validated germline DMRs (gDMRs), which usually function as imprinting control elements, were hypermethylated in 4F-iPSCs and hypomethylated in mESCs and C-iPSCs (Supplementary Figure 5).

Aberrant methylation in DMRs in the imprinted clusters can lead to diseases such as Prader-Willi syndrome (PWS), a complex neurogenetic disorder, caused by loss of expression of paternally imprinted genes located on human chromosome 15q11-q13 $3^{34,35}$. The PWS imprinted cluster in human has its orthologous locus on mouse chromosome 7C, that is, the Peg12-Ube3a cluster $^{36}$. In addition, multiple studies have identified the $5^{\prime}$ untranslated region of the Snrpn gene, which has been identified as the PWS Imprinting Center (IC).

Our data showed that all three DMRs within Peg12Ube $3 a$ cluster were hypermethylated in 4F-iPSCs (Fig. 5a) and consistently, their target genes Snhg14, Snurf/Snrpn, $N d n$, and Peg12 were silenced in 4F-iPSCs (Fig. 5b). In contrast, interestingly, the DMRs in Snrpn/Snurf loci were hypomethylated in C-iPSCs and mESCs (Supplementary Figure $3 \mathrm{~b}$ ) and therefore, the target genes were transcribed. Contrarily, expression level of another target gene 
a

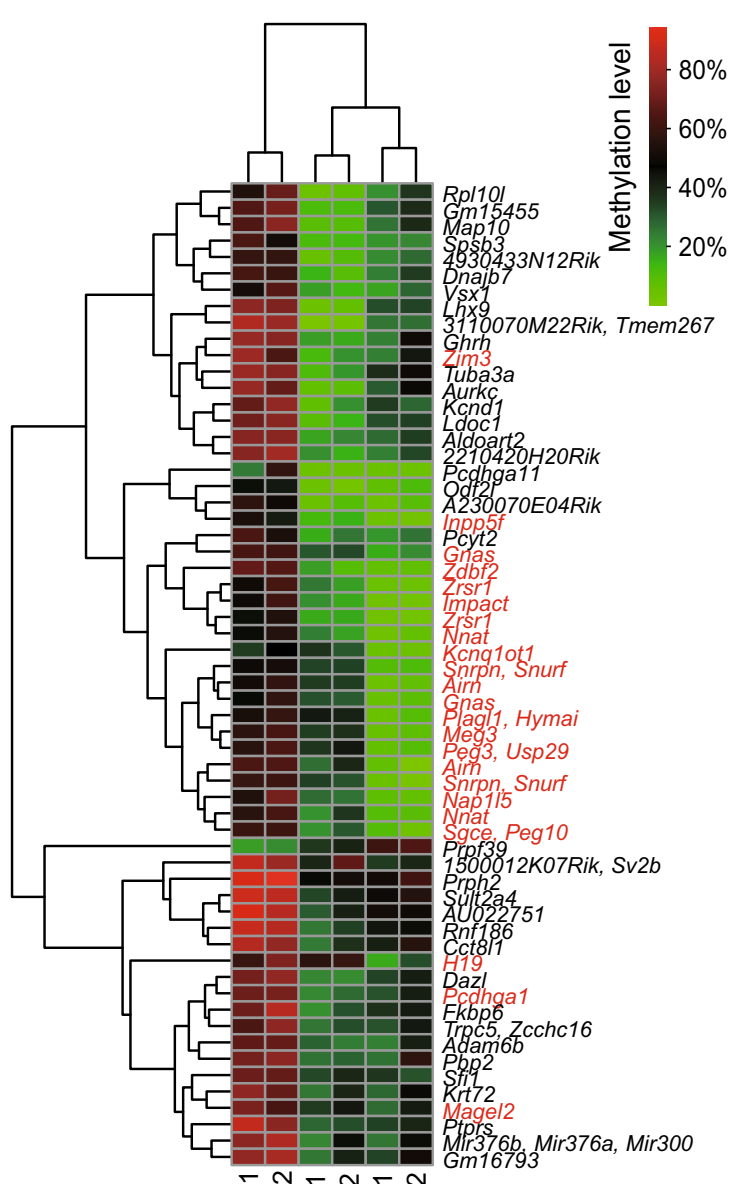

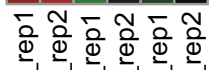

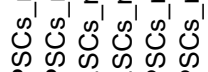

की

C

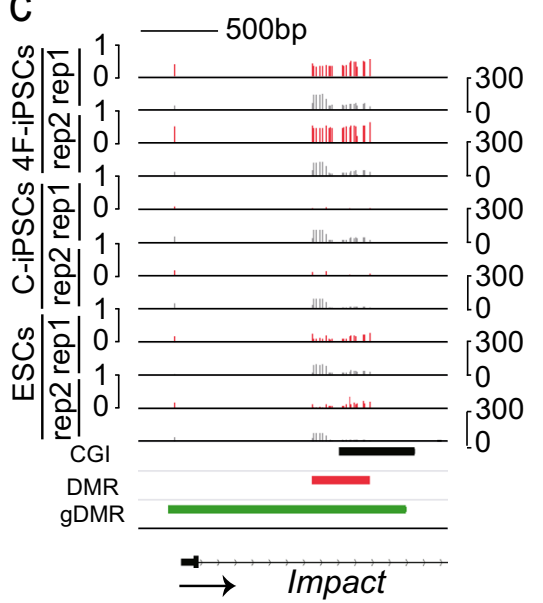

b

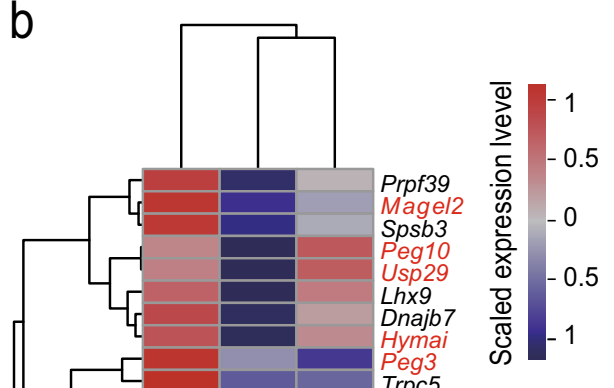

Impact

\section{d} 4F-iPSCs
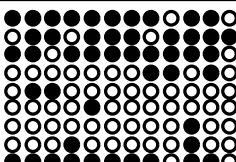

$40.83 \%$

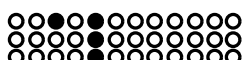

C-iPSCs

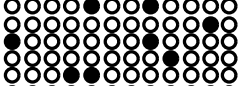

$10.83 \%$

ㅇํㅇำㅇํำ

mESCs

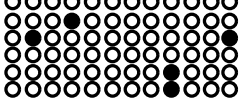

$10.83 \%$

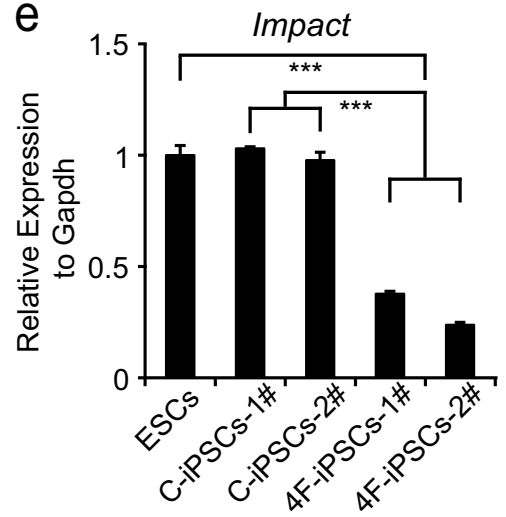

Fig. 4 (See legend on next page.) 
(see figure on previous page)

Fig. 4 The effects of DNA methylation in promoter DMRs on gene expression among C-iPSCs, 4F-iPSCs, and mESCs. a Hierarchical clustering of methylation in the promoter DMRs between 4F-iPSCs and C-iPSCS. The red fonts are imprinted genes. $\mathbf{b}$ Hierarchical clustering of the expression profiles of genes associated with the DMRs in $\mathbf{a}$. The red fonts are imprinted genes. $\mathbf{c}$ Representative DNA methylation profiles of the imprinted gene Impact. Red vertical lines are DNA methylation levels. Gray vertical lines are sequenced cytosine counts. CGls from UCSC genome browser, DMRs identified in this study, and the published germline DMRs (gDMRs) are shown at the bottom. The arrow indicates the transcription direction of Impact. d Bisulfite sequencing analysis of DMR within Impact as labeled in c. Each open and filled circle represents a methylated and non-methylated $\mathrm{CPG}$, respectively. The percentage of DNA methylation is shown. e Expression levels (qRT-PCR) of Impact in the three cell types. The data are presented as the mean \pm SEM of experiments performed in triplicate (*** $p$ value $<0.001$, Student's $t$ test)

Magel2 was positively correlated to methylation levels in the DMRs (Fig. 4a, b), implying a more complex regulatory system than just DNA methylation (Fig. 6).

\section{Discussion}

Collectively, our studies indicated that C-iPSCs were more hypomethylated than 4F-iPSCs and their DNA methylation status was closer to mESCs than to 4F-iPSCs. Notably, this may correlate with the different development potentials of $\mathrm{C}$-iPSCs and 4F-iPSCs, which are highlighted by the fact that $4 \mathrm{~F}-i \mathrm{PSCs}$ contribute to chimeras to a lesser extent $10-50 \%{ }^{19}$, while C-iPSCs generate chimeras as about $100 \%^{22}$. This reduced development potential of 4F-iPSCs may be also partly explained by abnormal silencing of the specific imprinted genes.

We also noted that repeat elements (LINEs, SINEs, and LTRs) in both C-iPSCs and 4F-iPSCs tend to be hypermethylated, and especially in 4F-iPSCs, in contrast to mESCs. This hypermethylation of DNA levels might be involved in the silencing of retrotransposons in C-iPSCs and $4 \mathrm{~F}-\mathrm{iPSCs}$. The role of repeat elements in pluripotency is complex and they can be both beneficial and deleterious $^{25}$, for example many long-non-coding RNAs are derived from repeat elements and are required to maintain pluripotency ${ }^{12,37}$. Reprogramming mimics early embryonic development, where retroelements of specific families show embryonic stage-specific demethylation before inner cell mass stage and then de novo DNA methylation occurs, while other elements remain higher methylation levels throughout development ${ }^{24}$. Unlike CiPSCs and mESCs, the methylation levels of repeat elements in 4F-iPSCs are somewhat similar to mouse somatic cells. The lack of correct DNA demethylation of repeat elements in 4F-iPSCs might imply an incomplete reprogramming.

Besides, our results showed that C-iPSCs and 4F-iPSCs have differences in the pattern of DNA methylation in non-coding regions (Fig. 1c). These non-coding regions may include promoters and distal regulatory elements, such as enhancers and transcription factor binding sites. Growing evidence suggests that cell- and tissue-specific changes in DNA methylation are associated with noncoding sequences ${ }^{38-41}$. Many studies have suggested that super-enhancers, which regions of the genome occupied by multiple transcription factors, cofactors, chromatin regulators, and transcription apparatus, are associated with the expression of key cell identity genes ${ }^{42,43}$ and many tissue-specific enhancers are hypomethylated in tissues where the target genes are expressed, but are hypermethylated in tissues where the target genes are silent ${ }^{40,41}$. Thus, we hypothesize that the differences in DNA methylation on non-coding regions may regulate the expression of target genes.

In addition, previous studies reported that human iPSCs created from PWS patients using the four pluripotency factors OCT4, SOX2, KLF4, and MYC retained the molecular signature of PWS including hypermethylation of SNRPN and $\mathrm{NDN}^{44}$. Mouse models lacking the Snrpn/ Snurf gene showed a postnatal lethality and surviving mice showed growth retardation similar to PWS phenotype $^{34 .}$ And the NDN gene encodes the protein necdin and Necdin-null mouse model showed respiratory defect that was also similar to those found in PWS patients ${ }^{34}$. Our findings suggest that 4F-iPSCs, although mostly similar to mESCs and C-iPSCs, have abnormal DNA methylation and expression at some key imprinted genes. Thus these differences may be not only derived from the original cells, but also a widespread phenomenon. The mechanisms that might cause this imprinting disorder require further studies.

Overall, this study is the first report, which demonstrates the DNA methylation profiles of C-iPSCs in a genome-wide scale, and compares the differential DNA methylation at imprinted regions among C-iPSCs, 4FiPSCs, and mESCs. Taken together, we provide a strong evidence that chemical reprogramming might be better than transcription factor-integrated reprogramming.

\section{Materials and Methods \\ Mice}

C57BL/6J, CBA/CaJ, and 129S4/SvJaeJ mice were purchased from the Jackson Laboratory. C57BL/6J, CBA/CaJ, and 129S4/SvJaeJ were used for generating OG2-MEFs. Animal experiments were performed according to the guidelines for the Care and Use of Laboratory Animals of the National Institutes of Health. 
a

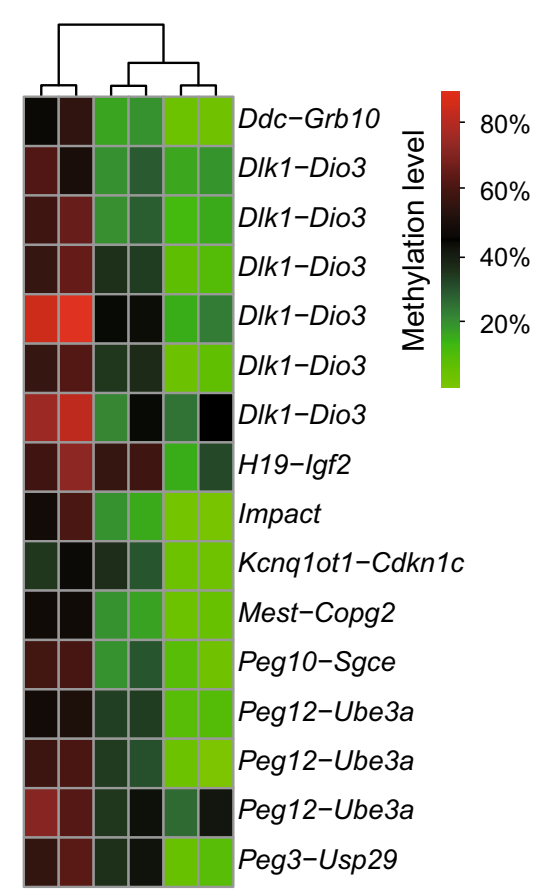

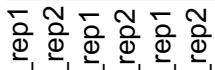

y'

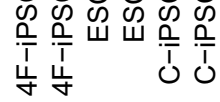

b

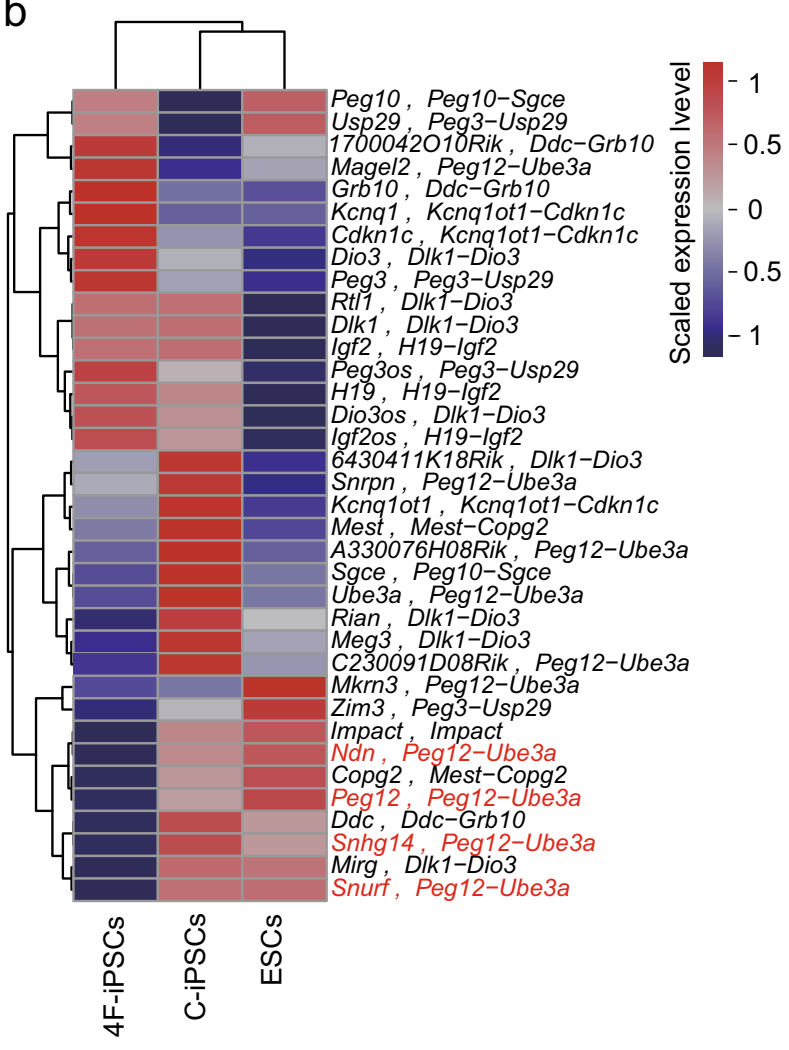

C

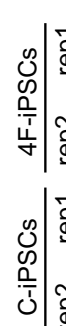

$5 \mathrm{~kb}$

峁

ช)

虫

$-5 \mathrm{~kb}$

d
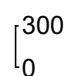

300

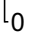

300
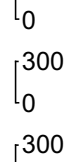

0

300

CG

DMR

gDMR

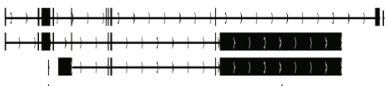

$\longrightarrow$ Meg3

IG-DMR

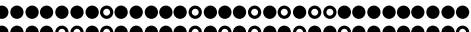
०0000000000000

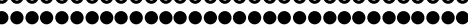

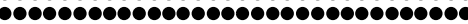
(.)०० 

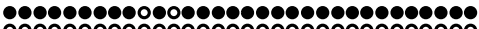
00000000000000000000000000000000 $74.06 \%$

00000000000000000000000000000000  0000000000000000000000000000000 C-iPSCs 00000000000000000000000000000000 (1)  00000000000000000000000000000000 -0000000000000000000000000000000 $36.25 \%$

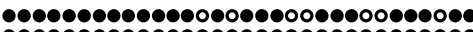


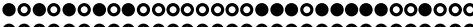
00000000000000000000000000000000 mESCs 000000 (1) 00000000000000000000000000000000 00000000000000000000000000000000

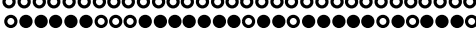
$27.81 \%$

Fig. 5 (See legend on next page.) 
(see figure on previous page)

Fig. 5 The effects of DNA methylation in DMRs within imprinting clusters on gene expression among C-iPSCs, 4F-iPSCs, and mESCs. a Hierarchical clustering of methylation levels in DMRs within imprinting regions between 4F-iPSCs and C-iPSCs. The names of imprinted gene clusters where DMRs locate are indicated on the right. $\mathbf{b}$ Hierarchical clustering of the expression profiles of the genes within the imprinted gene clusters. The names of the imprinted gene clusters where the genes are located are indicated after the comma. The four genes in red are used in the model schema (Fig. 6). c The methylation levels of imprinting control region near Meg3 locus. Red vertical lines are DNA methylation levels. Gray vertical lines are sequenced cytosine counts. CGIs from UCSC genome browser, DMRs identified in this study, and the published germline DMRs (gDMRs) are shown at the bottom. The arrow indicates the transcription direction of Meg3. $\mathbf{d}$ Bisulfite sequencing analysis of IG-DMR regulating expression of Meg3 and Mirg shown in Supplementary Figure 4. Each open and filled circle represents a methylated and non-methylated CpG, respectively. The percentage of DNA methylation is shown

\section{Cell culture}

Plat-E cells were maintained in DMEM high-glucose media (Hyclone) supplemented with 10\% FBS (Excell). OG2-MEFs were maintained in Dulbecco's high-glucose modified eagles medium (DMEM) containing 15\% fetal bovine serum (FBS, Gibco), $1 \mathrm{mM}$ non-essential amino acids (Gibco), 1\% GlutaMAX (Gibco). Mouse E14 ESCs, 4F-iPSCs, and C-iPSCs were maintained on gelatin-coated plates in a mESC culture medium containing DMEM high glucose (Gibco), 15\% FBS (Gibco), 1\% GlutaMAX (Gibco), $1 \%$ non-essential amino acids (NEAA, Gibco), $1 \mathrm{mM}$ sodium pyruvate (Gibco), 1\% penicillin-streptomycin (Gibco), $0.055 \mathrm{mM}$ 2-mercaptoethanol (Life Technologies), $1000 \mathrm{U} / \mathrm{ml}$ leukemia inhibitory factor (LIF,
Millipore), and supplemented with 2i (3 $\mu$ M CHIR99021 (Selleck) and $1 \mu \mathrm{M}$ PD0325901 (Selleck)).

\section{C-iPSCs generation}

Chemical iPSCs were generated from mouse OG2MEFs using small-molecule cocktails, as previously described $^{22}$. OG2-MEFs were plated 50,000 cells per well of a six-well plate. The next day (day 0 ), the culture was changed into stage 1 medium: knockOut DMEM (Gibco) supplemented with $10 \%$ KnockOut $^{\mathrm{TM}}$ serum replacement (KSR, Gibco), 10\% FBS (Gibco), 1\% GlutaMAX (Gibco), 1\% NEAA (Gibco), $0.055 \mathrm{mM}$ 2-mercaptoethanol (Life Technologies), $1 \%$ penicillin-streptomycin (Gibco), 100 $\mathrm{ng} / \mathrm{ml}$ bFGF (Origene) and the small-molecule cocktail

Mouse Chr7: PWS domains
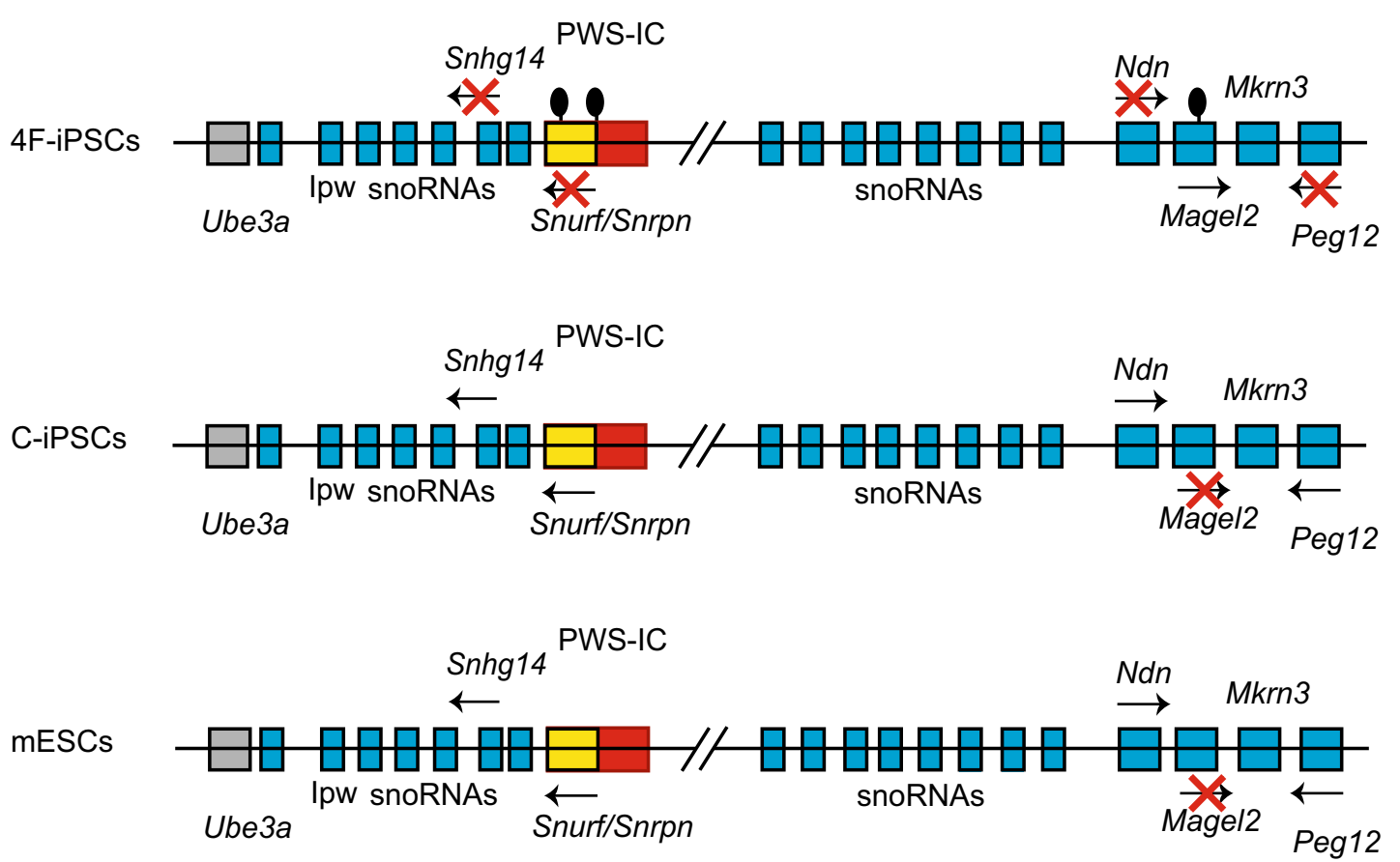

Fig. 6 Schematic diagrams of mouse Peg12-Ube3a imprinting cluster among 4F-iPSCs, C-iPSCs, and mESCs. 
VC6TFAE (0.5 mM VPA (Sigma), $20 \mu \mathrm{M}$ CHIR99021 (Selleck), $10 \mu \mathrm{M} 616452$ (Selleck), $5 \mu \mathrm{M}$ tranylcypromine hydrochloride (Tocris), $50 \mu \mathrm{M}$ Forskolin (Enzo Life Sciences), $0.05 \mu \mathrm{M}$ AM580 (Tocris), and $5 \mu \mathrm{M}$ EPZ004777 (Selleck)). On day 12, the cells were replated at 150,000 cells per well of a six-well plate. During days 12-16, concentrations of bFGF, CHIR, and forskolin were reduced to $25 \mathrm{ng} / \mathrm{ml}, 10 \mu \mathrm{M}$, and $10 \mu \mathrm{M}$, respectively. On day 16, the culture was changed into stage 2 medium containing $25 \mathrm{ng} / \mathrm{ml}$ bFGF, $0.5 \mathrm{mM}$ VPA, $10 \mathrm{mM}$ CHIR99021, $10 \mathrm{mM}$ 616452, $5 \mathrm{mM}$ tranylcypromine, 10 $\mathrm{mM}$ forskolin, $0.05 \mathrm{mM}$ AM580, $0.05 \mu \mathrm{M}$ DZNep (Tocris), $0.5 \mu \mathrm{M}$ 5-Aza-2'-deoxycytidine (Enzo Life Sciences), and $5 \mu \mathrm{M}$ SGC0946 (Selleck). On day 28, the culture was transferred into stage 3 medium: mix DMEM/ F12 (Invitrogen) and Neurobasal (Invitrogen) with a proportion of 1:1, 1\% N2 supplement (Invitrogen), 2\% B27 supplement (Invitrogen), 1\% GlutaMAX (Gibco), 1\% NEAA (Gibco), $0.055 \mathrm{mM}$ 2-mercaptoethanol (Life Technologies), 1\% penicillin-streptomycin (Gibco), $3 \mu \mathrm{M}$ CHIR99021 (Selleck), $1 \mu$ M PD0325901 (Selleck), and $1000 \mathrm{U} / \mathrm{ml}$ leukemia inhibitory factor (LIF, Millipore). After another 8-12 days, mESC-like and green fluorescent protein-positive C-iPSC colonies emerged and were then picked up for expansion and characterization. Any medium was changed every 4 days.

\section{F-iPSCs generation}

30,000 OG2-MEFs were plated in a six-well plate and then infected twice with retroviral supernatants generated with Plat-E cells. Plat-E cells were transfected pMAXsOct $4 /$ Sox $2 / \mathrm{Klf} 4 / \mathrm{c}-\mathrm{Myc}$ using modified polyethylenimine. OG2-MEFs were infected with equal volumes of the four supernatants of each OSKM transcription factor containing polybrene at a final concentration of $8 \mu \mathrm{g} / \mathrm{ml}$. Infected cells were cultured with mESC medium and renewed daily. Green fluorescent protein-positive 4FiPSCs colonies appeared about day 10 post infection and were picked up day 14 for expansion.

\section{Immunofluorescence}

Cells were washed twice with PBS and then fixed in 4\% paraformaldehyde at room temperature for $20 \mathrm{~min}$. After fixation, cells were treated with $0.3 \%$ Triton

$\mathrm{X}-100$ in PBS containing 10\% goat serum at room temperature for $15 \mathrm{~min}$. Cells were then incubated with primary antibodies at $4^{\circ} \mathrm{C}$ overnight. The primary antibody used for cell immunofluorescence was anti-NANOG (Novus Biologicals). And then the cells were washed for three times and were incubated with corresponding secondary antibody in a cassette at room temperature for $1 \mathrm{~h}$. Then the cells were washed for three times with PBS and then nuclei were stained with DAPI (Sigma). Images were captured with an inverted microscope (DMI4000, Leica Microsystems).

\section{Quantitative real-time PCR}

Total RNA was collected using the TRIzol reagent (MRC). Complemetary DNAs were synthesized from $1 \mu \mathrm{g}$ RNA by using First-Strand cDNA synthesis Kit (TOYOBO) with Oligo18 (dT) and Random Primer. PCR was carried out using SYBR green (Genstar) and performed on a CFX Real-Time System (Bio-Rad).

\section{Bisulfite sequencing}

DNA was extracted using TIANamp Genomic DNA Kit (TIANGEN). Bisulfite modification of the isolated DNA was performed using EpiTect Bisulfite Kit (QIAGEN). The bisulfite-modified DNA was amplified by PCR or nested PCR using TaKaRa EpiTaq ${ }^{\text {TM }}$ HS (for bisulfitetreated DNA) (Takara). To purify the PCR products, the DNA fragments were separated by electrophoresis using a $2 \%$ agarose gel. Then the bands were excised and purified with the TIANgel purification kit (TIANGEN). The PCR products were cloned into the pMD18-T Vector (Takara). Randomly picked 10 clones from each sample were sequenced.

\section{RNA-seq and data analysis}

RNA sequencing libraries were sequenced on an Illumina Hiseq 4000 platform and $150 \mathrm{bp}$ paired-end reads were generated. Sequencing reads were aligned to annotated mouse transcripts (mm10) using TopHat v2.0.13 ${ }^{45}$. High-quality mapped read pairs were retained for evaluation of gene expression using Cuffdiff v2.2.1 ${ }^{46}$ with default parameters. Expression levels (FPKM, fragments per kilobase per million mapped reads) for each gene were converted to a $Z$-score for hierarchical 1 clustering analysis. Genes with no expression in all three cell types were removed before plotting heatmaps.

\section{Reduced representative bisulfite sequencing and data analysis}

Chemical iPSCs, 4F-iPSCs, and mouse ESCs were collected. Genomic DNA was digested with MspI (NEB) and libraries were size-selected (170-370 bp) on an agarose gel followed by bisulfite conversion. Libraries were sequenced on an Illumina HiSeq 2500 sequencer as paired-end 50-bp reads.

Sequencing reads of RRBS were aligned to mouse reference genome (mm10) using BSMAP v2.90 ${ }^{47}$ with up to $8 \%$ mismatches. Only uniquely mapped pairs were retained for analysis of DNA methylation. MOABS v1.3.2 ${ }^{48}$ was used to merge replicates and measure CpG methylation levels. Only the CpGs with $\geq 10$-fold coverage in all samples were kept for differentially methylated CpG 
sites (DMCs) and DMR calling by mcall tool in MOABS with default parameters. Totally 1,106,981 CpGs were obtained. Strong hyper- or hypo-methylated (methylation differences $>33.3 \%, p$ value $<0.05$ ) DMCs and DMRs were retrieved from MOABS output for further analysis according to Fisher's exact test method.

Promoters were defined as -1 to $1 \mathrm{~kb}$ of the transcription start sites. Promoter classes based on $\mathrm{CpG}$ density (HCP, ICP, and LCP) were obtained by Bioconductor package compEpiTools v1.6.4 ${ }^{49}$ using R v3.3.0. HCP, ICP, and LCP were defined as previously published $^{50}$. Genomic locations of CpG islands and interspersed repeat families (LINEs, SINEs, LTRs) were downloaded from UCSC genome browser. The gDMRs were downloaded from: https://atlas.genetics.kcl.ac.uk/ $/^{51}$ and converted to the coordinates of mm10 genome assembly. The DMRs that are overlapped with a promoter are defined as promoter DMRs. Imprinting-region DMRs were defined in a similar manner.

Strong hypermethylated promoter DMRs in C-iPSCs that are at least $33.3 \%$ higher than mESCs were defined strong hypermethylated promoters DMRs in C-iPSCs compared to mESCs. The strong hypermethylated promoters DMRs in 4F-iPSCs compared to mESCs were defined in the same way. We merged the overlapped DMRs from these two sets of strong hypermethylated promoters DMRs and re-calculated the methylation level of the newly merged DMRs for each cell type. The methylation level of a DMR was calculated as the averaged methylation level of CpGs within the DMR.

In order to compare all DMRs between every two samples together, overlapped DMRs were merged and methylation ratio of merged DMRs were defined as the averaged methylation ratio of $\mathrm{CpGs}$ within the merged DMRs. We applied $K$-means $(K=3)$ method to clustering promoter DMRs into three classes. The genes in each cluster were re-ordered by official symbol.

\section{Statistical analysis}

Data are presented as mean values \pm SD unless otherwise indicated in figure legends. For statistical comparison of two groups, we performed two-tailed Student's $t$ test. Differences in means were considered statistically significant at $p<0.05$. Significance levels are: " $p<0.05$; "**" $p$ $<0.01 ; * * * 0.001$.

Comparisons between two CpGs or methylation regions were performed using Fisher's exact test $(p<0.05$ was considered significant).

\section{Accession numbers}

The RNA-seq and RRBS data sets have been deposited in the Gene Expression Omnibus (GEO) under accession number GSE92985. MEF RRBS data sets were downloaded from GEO under accession number GSE52741 ${ }^{15}$.

\section{Primers}

Primers for quantitative PCR

$\begin{array}{ll}\text { Primers } & \text { Sequence } \mathbf{( 5}^{\prime} \text {-3') } \\ \text { Oct4-forward } & \text { GGCTTCAGACTTCGCCTCC } \\ \text { Oct4-reverse } & \text { AACCTGAGGTCCACAGTATGC } \\ \text { Sox2-forward } & \text { AGGGCTGGGAGAAAGAAGA } \\ \text { Sox2-reverse } & \text { CCGCGATTGTTGTGATTAGT } \\ \text { Nanog-forward } & \text { AAGCAGAAGATGCGGACTGT } \\ \text { Nanog-reverse } & \text { ATCTGCTGGAGGCTGAGGTA } \\ \text { Impact-forward } & \text { GTGAAGAAATCGAAGCAATGGC } \\ \text { Impact-reverse } & \text { GGTACTCACTTGGCAACATCA } \\ \text { Snurf-forward } & \text { TCCAGGTCAAACGTCGAAGG } \\ \text { Snurf-reverse } & \text { CGTGGGTACAAGTGACACTCTT } \\ \text { Meg3-forward } & \text { TTGCACATTTCCTGTGGGAC } \\ \text { Meg3-reverse } & \text { AAGCACCATGAGCCACTAGG } \\ \text { Mirg-forward } & \text { GCGGTCAACACTGGGTACTT } \\ \text { Mirg-reverse } & \text { CCTGAGGACCAATTCAGCGT }\end{array}$

Primers for bisulphite sequencing analysis

\begin{tabular}{|c|c|}
\hline Primers & Sequence $\left(5^{\prime}-3^{\prime}\right)$ \\
\hline Nanog-forward & GATTTGTAGGTGGGATTAATTGTGAATTT \\
\hline Nanog-reverse & ACCAAAAAAACCCACACTCATATCAATATA \\
\hline Snrpn/Snurf-outside forward & TATGTAATATGATATAGTTTAGAAATTAG \\
\hline Snrpn/Snurf-outside reverse & AATAAACCCAAATCTAAAATATITTAATC \\
\hline Snrpn/Snurf-inside forward & AATTTGTGTGATGTTTGTAATTATTTGG \\
\hline Snrpn/Snurf-inside reverse & ATAAAATACACTTTCACTACTAAAATCC \\
\hline IG-DMR-outside forward & GTGTTAAGGTATATTATGTTAGTGTTAGG \\
\hline IG-DMR-inside forward & ATATTATGTTAGTGTTAGGAAGGATTGTG \\
\hline IG-DMR-reverse & TACAACCCTTCCСТСACTCCAAAAATT \\
\hline Impact-forward & TGGATGAGGTGTATAATTIT \\
\hline Impact-reverse & CAAAACAAAACTAAACCTAC \\
\hline
\end{tabular}

\section{Acknowledgements}

We thank Dr. Andrew Hutchins for critical reading and comments on this work and Dr. Hongkui Deng for helpful discussion. This work was supported by the Strategic Priority Research Program of the Chinese Academy of Sciences (XDA16010206), the Ministry of Science and Technology of the People's Republic of China (2015CB964800, 2016YFA0100400, and 2016YFA0100302), National Natural Science Foundation of China (31471210, 31601050, 31701131 $31771419,31721003,91519309$, and 91019017), Guangdong Frontier and Key Technology Innovation Special Grant (2016B030229006), Guangdong Natural Science Funds (2016A030313168, 2015A030308003, and 2015A030310041), Guangzhou Science Technology and Innovation Commission (201707020042), Foreign Science and Technology Cooperation Program of Guangzhou (20180704-HZ-0040), Science and Technology Planning Project of Guangdong Province, China (2017B030314056). We thank the support from the Guangzhou Branch of the Supercomputing Center of CAS. 


\section{Author details}

${ }^{1}$ CAS Key Laboratory of Regenerative Biology, Joint School of Life Sciences, CAS Center for Excellence in Molecular Cell Science, Guangzhou Institutes of Biomedicine and Health, Chinese Academy of Sciences, Guangzhou Medical University, Guangzhou, China. ${ }^{2}$ Institute of Health Sciences, Anhui University, Hefei, China. ${ }^{3}$ Guangdong Provincial Key Laboratory of Stem Cell and Regenerative Medicine, Guangzhou Institutes of Biomedicine and Health, Chinese Academy of Sciences, Guangzhou, China. ${ }^{4}$ The School of Life Sciences and Technology, Shanghai Key Laboratory of Signaling and Disease Research, The Collaborative Innovation Center for Brain Science, Tongji University, Shanghai, China

\section{Author contributions}

H.Y. and W.P. initiated the study and designed the experiments. W.P. conducted the experiments. J.H. and C.J. performed the bioinformatics analysis. G.H., Y.S., Q.X., M.Y., and S.G. contributed to the work. W.P., J.H., H.Y., and C.J. wrote the manuscript. H.Y. and C.J. conceived and supervised the entire study.

\section{Conflict of interest}

The authors declare that they have no conflict of interest.

\section{Publisher's note}

Springer Nature remains neutral with regard to jurisdictional claims in published maps and institutional affiliations.

Supplementary Information accompanies this paper at (https://doi.org/ 10.1038/s41419-017-0234-x).

Received: 1 October 2017 Revised: 16 November 2017 Accepted: 11 December 2017

Published online: 07 February 2018

\section{References}

1. Stadtfeld, M. \& Hochedlinger, K. Induced pluripotency: history, mechanisms, and applications. Genes Dev. 24, 2239-2263 (2010).

2. Gurdon, J. B. The developmental capacity of nuclei taken from intestinal epithelium cells of feeding tadpoles. J. Embryol. Exp. Morphol. 10, 622-640 (1962).

3. Takahashi, K. \& Yamanaka, S. Induction of pluripotent stem cells from mouse embryonic and adult fibroblast cultures by defined factors. Cell 126, 663-676 (2006).

4. Hou, P. et al. Pluripotent stem cells induced from mouse somatic cells by small-molecule compounds. Science 341, 651-654 (2013).

5. Cowan, C. A., Atienza, J., Melton, D. A. \& Eggan, K. Nuclear reprogramming of somatic cells after fusion with human embryonic stem cells. Science $\mathbf{3 0 9}$ 1369-1373 (2005).

6. Lister, R. et al. Hotspots of aberrant epigenomic reprogramming in human induced pluripotent stem cells. Nature 471, 68-73 (2011).

7. Planello, A. C. et al. Aberrant DNA methylation reprogramming during induced pluripotent stem cell generation is dependent on the choice of reprogramming factors. Cell Regen. 3, 4 (2014).

8. Miura, K. et al. Variation in the safety of induced pluripotent stem cell lines. Nat. Biotechnol. 27, 743-745 (2009).

9. Maherali, N. et al. Directly reprogrammed fibroblasts show global epigenetic remodeling and widespread tissue contribution. Cell Stem Cell 1, 55-70 (2007).

10. Wernig, M. et al. In vitro reprogramming of fibroblasts into a pluripotent EScell-like state. Nature 448, 318-324 (2007).

11. Zhao, B. et al. Genome-wide mapping of miRNAs expressed in embryonic stem cells and pluripotent stem cells generated by different reprogramming strategies. BMC Genomics 15, 488 (2014).

12. Loewer, $\mathrm{S}$. et al. Large intergenic non-coding RNA-RoR modulates reprogramming of human induced pluripotent stem cells. Nat. Genet. $\mathbf{4 2}$, 1113-1117 (2010).

13. Hussein, S. M. et al. Copy number variation and selection during reprogramming to pluripotency. Nature 471, 58-62 (2011).
14. Okita, K., Ichisaka, T. \& Yamanaka, S. Generation of germline-competent induced pluripotent stem cells. Nature 448, 313-317 (2007).

15. $\mathrm{Hu}, \mathrm{X}$. et al. Tet and TDG mediate DNA demethylation essential for mesenchymal-to-epithelial transition in somatic cell reprogramming. Cell Stem Cell 14, 512-522 (2014).

16. Gao, Y. et al. Replacement of Oct4 by Tet1 during iPSC induction reveals an important role of DNA methylation and hydroxymethylation in reprogramming. Cell Stem Cell 12, 453-469 (2013).

17. Doi, A. et al. Differential methylation of tissue- and cancer-specific CpG island shores distinguishes human induced pluripotent stem cells, embryonic stem cells and fibroblasts. Nat. Genet. 41, 1350-1353 (2009).

18. Kim, K. et al. Epigenetic memory in induced pluripotent stem cells. Nature 467, 285-290 (2010)

19. Stadtfeld, M. et al. Aberrant silencing of imprinted genes on chromosome 12qF1 in mouse induced pluripotent stem cells. Nature 465, 175-181 (2010).

20. Su, J. B., Pei, D. Q. \& Qin, B. M. Roles of small molecules in somatic cell reprogramming. Acta Pharmacol. Sin. 34, 719-724 (2013).

21. Stadtfeld, M. et al Ascorbic acid prevents loss of Dlk1-Dio3 imprinting and facilitates generation of all-iPS cell mice from terminally differentiated $B$ cells. Nat. Genet. 44, 398-405 (2012)

22. Hu, W. et al. Direct conversion of normal and alzheimer's disease human fibroblasts into neuronal cells by small molecules. Cell Stem Cell 17, 204-212 (2015).

23. Long, Y., Wang, M., Gu, H. \& Xie, X. Bromodeoxyuridine promotes full-chemical induction of mouse pluripotent stem cells. Cell Res. 25, 1171-1174 (2015).

24. Smith, Z. D. et al. A unique regulatory phase of DNA methylation in the early mammalian embryo. Nature 484, 339-344 (2012).

25. Hutchins, A. P. \& Pei, D. Transposable elements at the center of the crossroads between embryogenesis, embryonic stem cells, reprogramming, and long non-coding RNAs. Sci. Bull. 60, 1722-1733 (2015).

26. Wissing, $\mathrm{S}$. et al. Reprogramming somatic cells into iPS cells activates LINE-1 retroelement mobility. Hum. Mol. Genet. 21, 208-218 (2012).

27. Friedli, M. et al. Loss of transcriptional control over endogenous retroelements during reprogramming to pluripotency. Genome Res. 24, 1251-1259 (2014).

28. Roffe, M., Haij, G. N., Azevedo, H. F., Alves, V. S. \& Castilho, B. A. IMPACT is a developmentally regulated protein in neurons that opposes the eukaryotic initiation factor $2 a$ kinase GCN2 in the modulation of neurite outgrowth. J. Biol. Chem. 288, 10860-10869 (2013).

29. Lee Jeannie, T. \& Bartolomei Marisa, S. X-inactivation, imprinting, and long noncoding RNAs in health and disease. Cell 152, 1308-1323 (2013).

30. Plasschaert, R. N. \& Bartolomei, M. S. Genomic imprinting in development, growth, behavior and stem cells. Development 141, 1805-1813 (2014).

31. Edwards, C. A. \& Ferguson-Smith, A. C. Mechanisms regulating imprinted genes in clusters. Curr. Opin. Cell Biol. 19, 281-289 (2007).

32. Hiura, $H$. et al. DNA methylation imprints on the IG-DMR of the Dlk1-Gtl2 domain in mouse male germline. FEBS Lett. 581, 1255-1260 (2007).

33. Lin, S. P. et al. Asymmetric regulation of imprinting on the maternal and paternal chromosomes at the DIk1-Gtl2 imprinted cluster on mouse chromosome 12. Nat. Genet. 35, 97-102 (2003).

34. Bervini, S. \& Herzog, H. Mouse models of Prader-Willi syndrome: a systematic review. Front. Neuroendocrinol. 34, 107-119 (2013).

35. Nicholls, R. D., Saitoh, S. \& Horsthemke, B. Imprinting in Prader-Willi and Angelman syndromes. Trends Genet. 14, 194-200 (1998).

36. Brant, J. O., Riva, A., Resnick, J. L. \& Yang, T. P. Influence of the Prader-Willi syndrome imprinting center on the DNA methylation landscape in the mouse brain. Epigenetics 9, 1540-1556 (2014).

37. Ng, S. Y., Johnson, R. \& Stanton, L. W. Human long non-coding RNAs promote pluripotency and neuronal differentiation by association with chromatin modifiers and transcription factors. EMBO J. 31, 522-533 (2012).

38. Irizarry, R. et al. The human colon cancer methylome shows similar hypo- and hypermethylation at conserved tissue-specific CpG island shores. Nat. Genet. 41, 178-186 (2009).

39. Stelzer, Y., Shivalila Chikdu, S., Soldner, F., Markoulaki, S. \& Jaenisch, R. Tracing dynamic changes of DNA methylation at single-cell resolution. Cell 163 218-229 (2015).

40. Hon, G. C. et al. Epigenetic memory at embryonic enhancers identified in DNA methylation maps from adult mouse tissues. Nat. Genet. 45, 1198 (2013).

41. Heyn, H. et al. Epigenomic analysis detects aberrant super-enhancer DNA methylation in human cancer. Genome Biol. 17, 11 (2016)

42. Dowen, J. M. et al. Control of cell identity genes occurs in insulated neighborhoods in mammalian chromosomes. Cell 159, 374-387 (2014). 
43. Hnisz, D. et al. Super-enhancers in the control of cell identity and disease. Cell 155, 934-947 (2013).

44. Burnett, L. C. et al. Induced pluripotent stem cells (iPSC) created from skin fibroblasts of patients with Prader-Willi syndrome (PWS) retain the molecular signature of PWS. Stem Cell Res. 17, 526-530 (2016).

45. Kim, D. et al. TopHat2: accurate alignment of transcriptomes in the presence of insertions, deletions and gene fusions. Genome Biol. 14, R36 (2013).

46. Trapnell, C. et al. Differential analysis of gene regulation at transcript resolution with RNA-seq. Nat. Biotechnol. 31, 46-53 (2013).

47. Xi, Y. \& Li, W. BSMAP: whole genome bisulfite sequence MAPping program. BMC Bioinformatics 10, 232 (2009).
48. Sun, D. et al. MOABS: model based analysis of bisulfite sequencing data Genome Biol. 15, R38 (2014).

49. Kishore, K. et al. methylPipe and compEpiTools: a suite of R packages for the integrative analysis of epigenomics data. BMC Bioinformatics 16, 313 (2015).

50. Weber, M. et al. Distribution, silencing potential and evolutionary impact of promoter DNA methylation in the human genome. Nat. Genet. 39, 457-466 (2007).

51. Schulz, R. et al. WAMIDEX - a web atlas of murine genomic imprinting and differential expression. Epigenetics 3, 89-96 (2008). 